\title{
Broadband THz Generation from Photoconductive Antenna
}

\author{
Qing Chang ${ }^{1}$, Dongxiao Yang ${ }^{1,2}$, and Liang Wang ${ }^{1}$ \\ ${ }^{1}$ Zhejiang University, China \\ 2 The Electromagnetics Academy at Zhejiang University, China
}

\begin{abstract}
Since the observation of radiation in the Terahertz (THz) frequency range from photoconductive antennas exited by femtosecond ( $\mathrm{fs}$ ) laser pulses, considerable efforts have been made to understand the mechanism responsible for THz generation, and to develop applications in THz Time-Domain Spectroscopy (THz-TDS). In this paper, the calculation of $\mathrm{THz}$ radiation from biased photoconductive antenna is reported, given some amelioration of the calculation model and classical Drude-Lorentz theory. Some simulation results based on the new calculation model are shown.
\end{abstract}

\section{Introduction}

Rapid photoconductors have been used in the last two decades to generate ultrashort electrical signals with duration of several hundreds fs that have a spectrum situated in the $\mathrm{THz}$ region. Nowadays, this is the most popular method to generate/detect $\mathrm{THz}$ fields.

When a fs laser pulse with an arbitrary intensity profile $I(t)$ excites a biased semiconductor with photon energy greater than its bandgap, electrons and holes are produced at the illumination point in conduction and valence bands, respectively. The rapid changes of the photocarriers' density and their acceleration due to the applied DC bias Vb produce an electromagnetic field radiation into free-space with the help of an antenna. The production of ultrashort currents with a FWHM lifetime of 1 ps or less strongly depends on the carrier's lifetime in the semiconductor. Low temperature grown GaAs (LT-GaAs), with the short carrier lifetime $\left(\tau_{e}=0.1 \mathrm{ps}\right.$, $\tau_{h}=0.4 \mathrm{ps}$ ), is the most popular material. Among many materials, it is very suitable for the theory of $\mathrm{THz}$ Electromagnetic field radiation $[1,2]$.

The space-charge screening and radiation-field screening are two factors preventing photoconductor from generating higher power $\mathrm{THz}$ radiation, which could affect all kinds of the photoconductive antenna more or less. Based on the shape of antenna, their effects are different. Generally speaking, the space-charge screening can be important to small dipole antennas, but, for large area antennas, radiation-field screening is the major cause of saturation [3].

\section{Theory of the THz Wave Radiation from Photoconductive Antenna}

\subsection{Small Dipole Aperture (Drude-Lorentz Theory)}

In LT-GaAs, the trapping time of photo carriers is much shorter than the recombination time of electrons and holes, the carrier lifetime in LT-GaAs can be approximated as the carrier trapping time. The relationship of the free carriers and time is as follows [1]

$$
d n / d t=-n / \tau_{t}+G(t)
$$

where $n$ is the density of the carriers, $G(t)=n_{0} \exp (t / \Delta t)^{2}$ is the generation rate of the carriers after the laser pulse excitation, $\Delta t$ is the excitation time of the laser pulse, which is usually between 30 and $150 f s, n_{0}$ is the initial density of the carriers, $\tau_{t}$ is the trapping time of the carriers which depends on the growth temperature of the LT-GaAs and other factors. While in the biased field, the velocity rate of carriers can be written as follows

$$
d v_{e, h} / d t=-v_{e, h} / \tau_{r e l}+E \cdot q_{e, h} / m_{e, h}
$$

where $v_{e, h}$ is the average velocity of the carriers $q_{e, h}$ is the coulomb of the carriers $\tau_{r e l}$ is the momentum releasing time, which is about $30 \mathrm{fs}$ in LT-GaAs. $E$ is the local electric field, which can be described in this way

$$
E=E_{b}-P / \alpha \cdot \epsilon_{r}
$$


where $E_{b}$ is the biased electric field $\alpha$ is the geometric factor of the semiconductorwhich is equal to 3 in the case of LT-GaAs [2], $\epsilon_{r}$ is the dielectric constant $P$ is the polarization caused by the separation of the electron and the hole. The relationship of the polarization and time is as follows

$$
d P / d t=-P / \tau_{r e c}+J(t)
$$

where $\tau_{\text {rec }}$ is the time of the recombination time of electron and hole. $J$ is the current density on the surface of the semiconductor defined as follows

$$
J(t)=e n v_{h}-e n v_{e}
$$

Based on the Maxwell Equations, the $\mathrm{THz}$ radiation field in the far fields can be expressed as [3]

$$
E_{r}(z, t)=-\left[A /\left(4 \pi \epsilon_{0} C^{2} z\right)\right] \cdot d J(t) / d t
$$

where $A$ is the area of gap space. Based on EQ.1-6, we can get the far-field radiation expression

$$
E_{T H z} \propto \partial J / \partial t \propto e v \cdot \partial n / \partial t+e n \cdot \partial v / \partial t
$$

\subsection{Large Area Antenna}

The small dipole aperture antenna provides a normal way for $\mathrm{THz}$ radiation. However, because of the effect of space-charge screening, caused by the separation of the electron and hole (EQ.3), the polarized $P$ will be saturated, which will lead to the saturation of the radiation wave field. In order to solve this problem, the antenna with large area is widely used recently. Taylor has found the relationship of biased electric field and position [3]

$$
\partial E(x, t) / \partial x=e \cdot[p(x, t)-n(x, t)] / l \epsilon
$$

Based on this equation, when $l$, the size of the aperture between the two tips of the antenna, is larger than the wavelength of the radiation wave, $\partial E(x, t) / \partial x$ can be regarded as zero, which means the density of the electric field has no change in the whole area of the aperture. Meanwhile, because the aperture of the antenna is larger than the wavelength of the radiation wave, the carriers can not reach the ends of the antenna tip after an integrate $\mathrm{THz}$ wave sending out, so in the field around the antenna tip, the current density can be regarded as zero, and $E$ is equal to $E_{b}$. Consequently, based on EQ. 8, $E$ is equal to $E_{b}$ approximately, and in the whole area of aperture as well. In Reference [3], Taylor explained that even in the antenna with a large area, the polarization of the electron and hole (space-charge screening) has week effect on the THz radiation wave.

In photoconductive antenna with a large area aperture, the space-charge screening has little effect on the $\mathrm{THz}$ wave radiation, but there is another factor limiting the radiation power, radiation-field screening. Taylor has accurately compared those two effects according to experiments and calculation in [3].

\section{Amelioration of the Calculation Model}

In most previous papers, the calculation of the carriers' density is usually according to EQ.1, which is based on two hypotheses:

1) The $f s$ laser pulse is totally absorbed in the epitaxial layer of LT-GaAs.

2) The amplitudes of $f s$ laser pulse are identical at every position on the area of irradiation point at the same time.

If the two hypotheses base on the case that the power of the incident light is low and the aperture area of the antenna is small, they can be perfect. Nevertheless, if the energy of the incident laser is high or the aperture area of the antenna is large, the error caused by these hypotheses will be insupportableness. Here, we introduce another way to calculate carrier density.

LT-GaAs is the epitaxial layer growing on GaAs substrate. But GaAs substrate layer does not have short carriers life time. On one side, we should make sure that the epitaxial layer is thick enough to absorb at least 95\% incident laser; on the other side, the thickness of the epitaxial layer can not be increased with no-limitation. So when the high-power laser illuminates on the LT-GaAs, the effect of the carrier with a long life time must be considered. In the epitaxial layer:

$$
\partial n(x, t) / \partial t=-[(1-R) / h v] \cdot \partial I_{o p t}(x, t) / \partial x-n(x, t) / \tau_{r, e p i}
$$


where $\tau_{r, \text { epi }}$ is the trapping time of the carriers in epitaxial layer, $I_{o p t}(x, t)$ is an intensity profile of laser pulse, $R$ is the intensity reflectivity of the surface; $x$ is the distance from the semiconductor surface to the measure point. The first term on the right side of the equation means the absorption of the incident laser, which is corresponded to the generation rate of electrons and holes, and the second one means that the electrons are captured or the electrons and the holes are recombined. If we suppose that the thickness of the extended layer is $l$, the density of the electrons at certain time $t$, can be written as:

$$
n_{e p i}=\int_{0}^{l} n(x, t) d x
$$

Thus, EQ.9 can be rewritten as

$$
\partial n_{e p i}(t) \partial t=(1-R) \cdot\left\{I_{o p t}(0, t)-I_{o p t}(l, t)\right\} / h v-n_{e p i}(t) / \tau_{r, e p i}
$$

In the same way, in the substrate layer, we can get the equations

$$
\begin{gathered}
n_{\text {sub }}=\int_{l}^{\infty} n(x, t) d x \\
\partial n_{\text {sub }}(t) / \partial t=(1-R) \cdot I_{\text {opt }}(l, t) / h v-n_{\text {sub }}(t) / \tau_{r, s u b}
\end{gathered}
$$

\section{Simulation and Discussion}

Based on the ameliorated calculation model, we will do some simulation based on some varied factor, which is concerned with the generation of the $\mathrm{THz}$ radiation, and analyze the effects of different factors to $\mathrm{THz}$ radiation.

\subsection{Trapping Time of Carriers}

Trapping time of carriers is one of the most important factors affecting the generation of $\mathrm{THz}$ wave. If the trapping time of carriers is not short enough, it will not radiate out electromagnetic wave

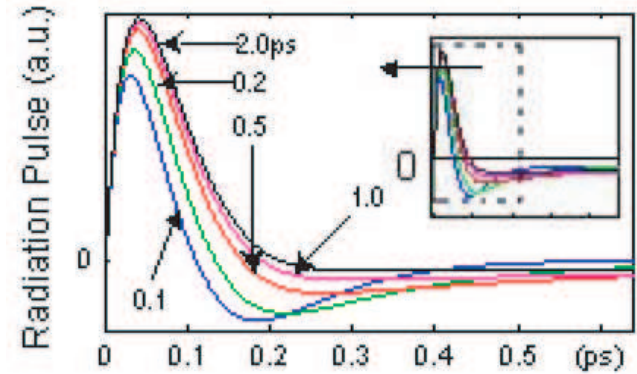

Figure 1: THz radiation pulses with different trapping time of $0.1,0.2,0.5,1.0$, and $2.0 \mathrm{ps}$, respectively.

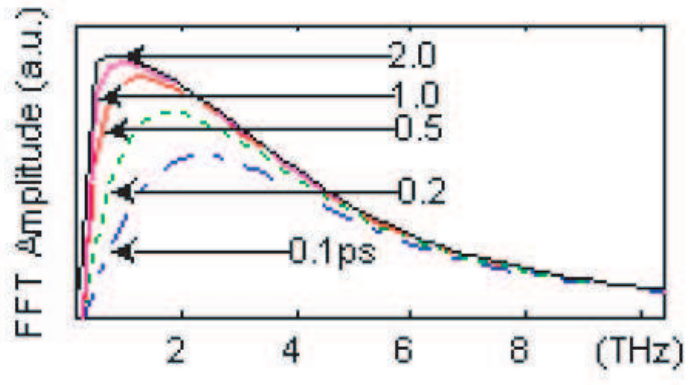

Figure 2: Fourier transformed spectra of radiation pulses with different trapping time.

in $\mathrm{THz}$ domain. In this simulation, the incident fs laser at the wavelength of $1550 \mathrm{~nm}$ and the pulse width of $150 \mathrm{fs}$ is used, the bias electric field is $300 \mathrm{~V}$, the space between the antenna tips is $5 * 5 \mathrm{um}$, and the separation between the emitter and the detector is $2 \mathrm{~mm}$. The semiconductor material is LT-GaAs with $m_{e}=0.067 m_{0}$ and $m_{h}=0.45 m_{0}\left(m_{e}\right.$ and $m_{h}$ are effective mass of electron and hole, respectively). The trapping time of carriers are varying based on the growth temperature of LT-GaAs and some other factors. Here, different carriers' trapping time of $0.1,0.2,0.5,1.0$, and 2.0 ps are simulated respectively, and shown in Fig.1. Figure 2 shows the corresponding FFT spectra.

The two figures show that LT-GaAs antenna with longer trapping time can generate higher power $\mathrm{THz}$ radiation.

\subsection{The Effect of the Laser Pulse and Duration}

The laser pulse is another primary factor in the generation of the $\mathrm{THz}$ wave. Different wavelength and pulse width of pump laser will have different effects on $\mathrm{THz}$ radiation. Figure 3 shows the wave shapes of the $\mathrm{THz}$ radiation, excited by the laser pulse with wavelength of $620 \mathrm{~nm}$ and $1550 \mathrm{~nm}$. Figure 4 shows the FFT spectra of the $\mathrm{THz}$ radiation wave, with the laser pulse width of $50 \mathrm{fs}, 100 \mathrm{fs}$, and $150 \mathrm{fs}$. In these simulations, trapping time of the carriers is $0.5 \mathrm{ps}$, and the other factors are the same as those in section 3.1. 
The longer pulse duration and the smaller wavelength of laser, the larger power each single laser pulse will have, which will generate more powerful THz wave. Additionally, from Fig.4, it is noted that although the 150 $f s$ laser pulse can generate the highest power $\mathrm{THz}$ wave, the $50 \mathrm{fs}$ laser generates the highest radiation power at higher frequency, such as that $5 \mathrm{THz}$ in Fig.4.

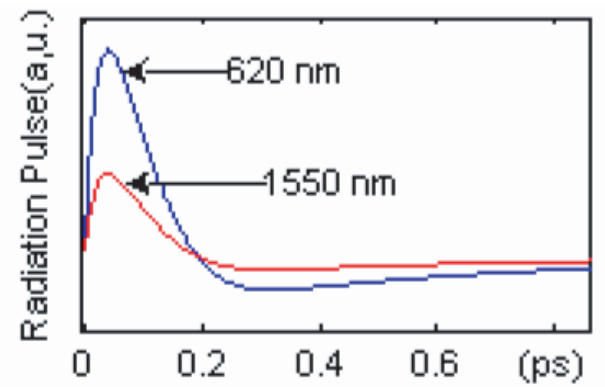

Figure 3: Radiation pulse with the laser pulse wavelength of $620 \mathrm{~nm}$ and $1550 \mathrm{~nm}$.

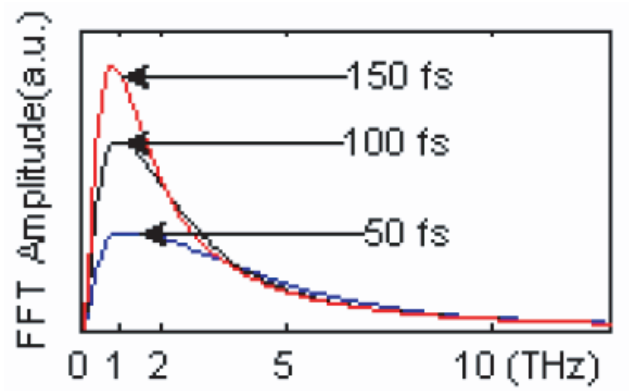

Figure 4: The FFT spectrum of the $\mathrm{THz}$ radiation with pulse width of 50, 100 and 150 fs, respectively.

\subsection{The Effect of the Bias Electric Field and Dipole Aperture Size}

In this simulation, a small dipole aperture is used, and the effect of space-charge screening is neglected. Figure 5 shows the time-domain pulses of the $\mathrm{THz}$ radiation waves, with the bias electric field of $200 \mathrm{~V}, 300 \mathrm{~V}$

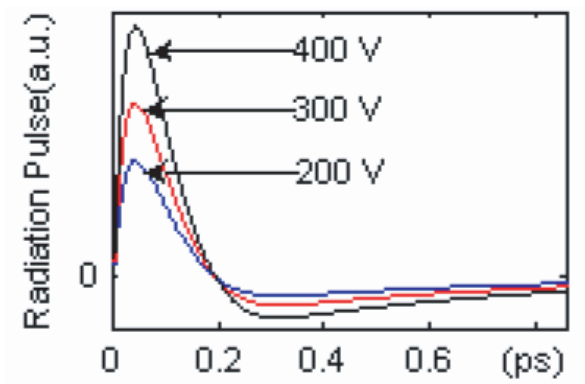

Figure 5: The time-domain pulse of the $\mathrm{THz}$ radiation wave, the bias electric field is $200 \mathrm{~V}, 300 \mathrm{~V}$ and $400 \mathrm{~V}$, respectively.

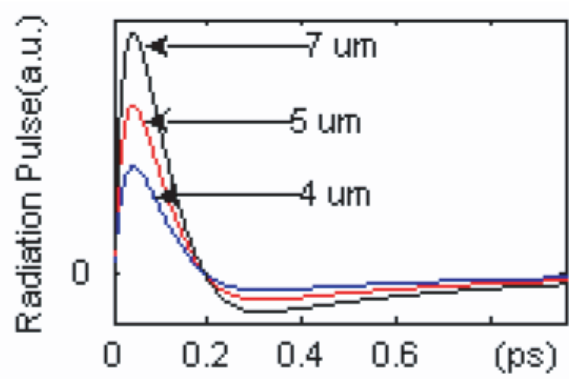

Figure 6: The time-domain pulse of the $\mathrm{THz}$ radiation wave, the separation is $4 \mathrm{um}, 5 \mathrm{um}$ and $7 \mathrm{um}$, respectively.

and $400 \mathrm{~V}$, respectively. Figure 6 shows the time-domain pulses of the $\mathrm{THz}$ radiation waves, with the separation between antennae of $4 \mu \mathrm{m}, 5 \mu \mathrm{m}$ and $7 \mu \mathrm{m}$, respectively. The trapping time of carriers is $0.5 \mathrm{ps}$, other conditions are the same as those in section 3.1 .

Based on the result of calculation, when the biased electric voltage are $200 \mathrm{~V}, 300 \mathrm{~V}$ and $400 \mathrm{~V}$, the peakto-peak ratios are 6.0808,6.0857 and 6.0908, respectively; when the separation aperture between the antennae are $4 \mu \mathrm{m}, 5 \mu \mathrm{m}$ and $7 \mu \mathrm{m}$, the peak-to-peak ratios are 6.0926,6.0921 and 6.0907, respectively.

According to the result of the calculation above, the conclusion can be made that if the effect of screening is neglected, the longer trapping time of the carriers, the lower energies of the incident laser pulse, the larger biased electrical voltage, and the less separation between the antennae, $\mathrm{THz}$ radiation would be more likely a single polar wave. In this case, it is determined by the lifetime of electron-hole pairs in LT-GaAs.

\section{Conclusion}

In this paper, an improvement for classical Drude-Lorentz theory model is discussed. The calculation results depending on the improvement are more likely precise. The calculated results show that THz radiation is mostly due to the fast change of carrier density, the laser pulse power and duration, and also the bias electric field and dipole aperture size.

\section{Acknowledgement}

This work was supported by NSFC under Grant No. 60477033. D.X. Yang's e-mail address is yangdx@zju.edu.cn 


\section{REFERENCES}

1. Piao, Zhisheng, Masahiko TaNI and Kiyomi SAKAI, "Carrier Dynamics and Terahertz Radiation In Photoconductive Antennas", Jpn. J. Appl. Phys., Vol. 39, 96-100, 2000.

2. Dragoman, D., M. Dragoman, "Terahertz Fields and Applications", Progress in Quantum Electronics, Vol. 28, 1 66, 2004.

3. Rodrigrez, G., A. J. Taylor, "Screening of the Bias Field in Terahertz Generation from Photoconductors", Optics Letters, Vol. 21, No. 14, July 15, 1996.

4. Peter, R. Smith, David H. Auston, "Subpicosecond Photoconducting Dipole Antennas", IEEE JOURNAL OF QUANTUM ELECTRONICS, Vol. 24, NO. 4, 1988. 